\title{
Near Real-time Surveillance of Disease during 2016-17 Influenza Season in the U.S.
}

\author{
Sushruth K. Reddy, Jhobe Steadman and John Tamerius*
}

Quidel Corporation, San Diego, CA, USA

\section{Objective}

Demonstrate performance of the Virena Global Wireless Surveillance System, an automated platform utilized in conjunction with the Sofia FIA Analyzer, for near real-time transmission of infectious disease test results to public health and other healthcare organizations.

\section{Introduction}

Public health agencies worldwide all enjoy the same missionproviding healthcare warnings, guidance, and support to the public and healthcare professionals they represent. A critical element in achieving this mission is accessing timely and comprehensive surveillance information about disease in their regions of responsibility. Advances in diagnostic technologies for infectious disease and in the wireless conveyance of information hold great promise for advancing the quality of surveillance information and in facilitating the delivery of timely, accurate, and impactful public health information. Quidel Corporation has developed a cloud-based, wireless communications system that is fully integrated with its Sofia fluorescence immunoassay (FIA) platform for rapid, point-of-care diagnosis of infectious disease. The system, called the Virena Global Wireless Surveillance System (hereinafter, Virena) provides test results to public health organizations and other appropriate entities in near-real time. Currently, more than 4,000 Sofia instruments are transmitting results automatically by Virena. This presentation describes the use of Virena in surveilling influenza in the U.S. in the 2016-2017 influenza season, when over 700,000 influenza-likeillness (ILI) patient results were transmitted. The methods employed, results, and the promise of this innovative system will be discussed.

\section{Methods}

The Sofia Fluorescent Immunoassay Analyzer (FIA) is a small FDA-cleared, CLIA-waived bench top device that uses immunofluorescence-based, lateral-flow technology for rapid analyte detection within 15 minutes for influenza. With Sofia2, a recent upgrade, positive influenza test results can be obtained in as few as 3 minutes, depending on virus levels. The results are encrypted, and automatically transmitted by Virena--often within 5 seconds-to a dual cloud system for further encryption and formatting. The test results (also including location, date, and patient age) are subsequently pushed to participating public health and healthcare organizations for daily collection and analysis. Healthcare providers utilizing the Virena system may also access their own data and facility-de-identified regional and national data, using a passwordenabled internet application called MyVirena.com.

\section{Results}

Between August 1, 2016 and October 6, 2017, 706,654 ILI patient results were transmitted by Virena from over 3,000 clinical sites in the United States. The influenza positivity rate (influenza A and B combined) peaked on February 9th at $33 \%$ and maintained this level for two weeks (Figure 1). During this period, as many as 7,048 results were transmitted by Virena per day. Influenza A activity peaked on the same day at $26 \%$, and influenza B peaked at $18 \%$ nearly 6.5 weeks later. In the six months from December $15^{\text {th }}$ to June $15^{\text {th }}$, the influenza positivity rate for patients with ILI was $10 \%$ or greater in the United States. Data analysis for individual states revealed significant differences in time of onset of influenza and in the peak positivity rates. For example, the state of Arizona experienced peak positivity rates for influenza activity ( $42 \%)$ as late as mid-May, driven largely by influenza B. In California, influenza A peaked at $43 \%$ on January $16^{\text {th }}$ and maintained a positivity rate greater than $15 \%$ for nearly three months, while influenza B averaged below $4 \%$ for the entire period. Age-specific analysis showed that children in the 2 to 18 year old group had the highest positivity rate $(44 \%, n=251,756)$ and the longest incidence period. Virena data demonstrated similar influenza activity trends on national and regional levels as that depicted by the clinical laboratory and NREVSS data collected by the CDC; however, the Virena data were collected and reported sooner (Figure 2).

\section{Conclusions}

The Virena system represents a major stride for disease surveillance, providing clinical testing data in near real-time time, with local, national, and global scope. This first substantial evaluation of the Virena system, with over 4,000 transmitting Sofia Analyzers, demonstrates capabilities for near real-time assessment of disease onset, regionally varying positivity rates, durations of outbreaks, differential assessment of influenza A and B prevalence, and dynamic mapping throughout the year. With expanding regional and metropolitan coverage, the Virena system holds promise as both a powerful surveillance tool, and as a valuable resource for healthcare quality initiatives, epidemiological research, and the development of new mathematical models for influenza forecasting.

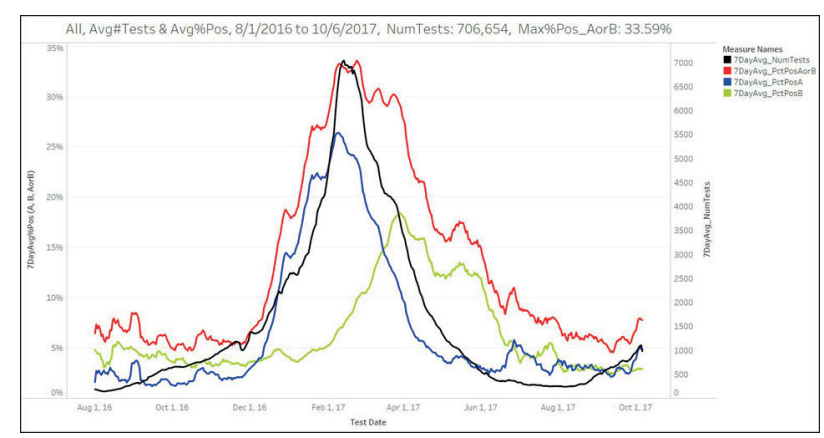

Figure 1: Influenza Status in U.S.A. by Virena 9-1-16 to 10-6-17 


\section{ISDS 2018 Conference Abstracts}

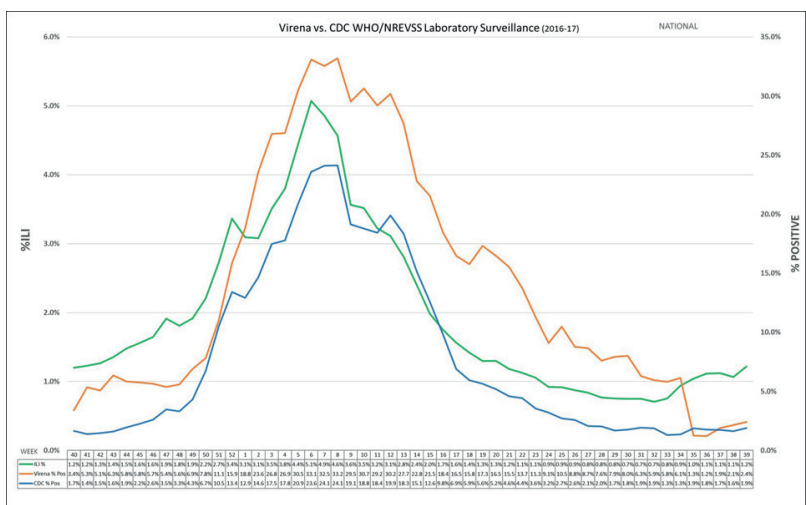

Figure 2: Comparison of Virena and CDC ILI and NREVSS Results 9-1-16 to $10-6-17$

\section{Keywords}

Surveillance; Influenza; Infectious Disease; Pandemic

\section{*John Tamerius}

E-mail: johntamerius@quidel.com 